\title{
Comparison of Hospital Consumer Assessment of Healthcare Providers and Systems Patient Satisfaction Scores for Specialty Hospitals and General Medical Hospitals: Confounding Effect of Survey Response Rate
}

\author{
Zishan K. Siddiqui, MD*, Albert W. Wu, MD, MPH'1,2, Nargiza Kurbanova ${ }^{3}$, Rehan Qayyum, MD, MHS
}

${ }^{1}$ Department of Medicine, Johns Hopkins University, Baltimore, Maryland; '2Department of Health Policy and Management, Johns Hopkins Bloomberg School of Public Health, Baltimore, Maryland; ${ }^{3}$ School of Health Professions-Nursing, The Community College of Baltimore County, Catonsville, Maryland.

BACKGROUND: Specialty hospitals are a subset of acutecare hospitals that provide a narrower set of services than general medical hospitals (GMHs), predominantly in areas such as cardiac disease and surgery. Although specialty hospitals also advertise high patient satisfaction, this has not been examined using national data. We examined the differences in Hospital Consumer Assessment of Healthcare Providers and Systems (HCAHPS) satisfaction scores in a national sample.

METHODS: HCAHPS results were obtained for July 2007 to June 2010. Specialty hospitals were identified using the American Hospital Association's Annual Survey, the Physician Hospital Association's directory, a name search of hospitals on the HCAHPS database, contact with experts, and online searches. Multiple linear regression was performed to examine the relationship between overall satisfaction and hospital specialty status, survey response rate, and subdomains of patient satisfaction.
RESULTS: We identified 188 specialty hospitals and 4368 GMHs. Specialty hospitals were disproportionately located in states that do not require Certification Of Need (47.9\%), and had a higher overall patient satisfaction score (86.6 vs $67.8 \%, P<0.0001)$ and survey response rates $(49.6 \%$ vs $32.2 \%, P<0.0001)$. After adjusting for response rate, the difference in overall patient satisfaction decreased by $>50 \%$ (from 18.5 to 8.7 ) but remained significantly higher $(P<0.0001)$. Similar results were obtained for patient satisfaction subdomains.

CONCLUSION: Specialty hospitals have a significantly higher overall HCAHPS patient satisfaction score than GMHs, although more than half of this difference disappears when adjusted for survey response rate. Comparisons among healthcare organizations should take into account survey response rates. Journal of Hospital Medicine 2014;9:590-593. (c) 2014 Society of Hospital Medicine
Patient satisfaction surveys are widely used to empower patients to voice their concerns and point out areas of deficiency or excellence in the patientphysician partnership and in the delivery of healthcare services. ${ }^{1}$ In 2002, the Centers for Medicare and Medicaid Service (CMS) led an initiative to develop the Hospital Consumer Assessment of Healthcare Providers and Systems (HCAHPS) survey questionnaire. ${ }^{2}$ This survey is sent to a randomly selected subset of patients after hospital discharge. The HCAHPS instrument assesses patient ratings of physician communication, nursing communication, pain control, responsiveness, room cleanliness and quietness, discharge process, and overall satisfaction. Over 4500 acute-care facilities routinely use this survey. ${ }^{3}$ HCAHPS scores are publicly reported, and patients can utilize these scores to compare hospitals and

\footnotetext{
*Address for correspondence and reprint requests: Zishan K. Siddiqui, MD, Assistant in Medicine, Hospitalist Program, Johns Hopkins School of Medicine, 600 N. Wolfe St., Room Nelson 223, Baltimore, MD 21287; Telephone: 443-287-3631; Fax: 410-502-0923; E-mail: zsiddiq1@jhmi.edu

Additional Supporting Information may be found in the online version of this article.

Received: September 6, 2013; Revised: April 28, 2014; Accepted: May 7, 2014

2014 Society of Hospital Medicine DOI 10.1002/jhm.2225

Published online in Wiley Online Library (Wileyonlinelibrary.com).
}

make informed choices about where to get care. At an institutional level, scores are used as a tool to identify and improve deficiencies in care delivery. Additionally, HCAHPS survey data results have been analyzed in numerous research studies. ${ }^{4-6}$

Specialty hospitals are a subset of acute-care hospitals that provide a narrower set of services than general medical hospitals (GMHs), predominantly in a few specialty areas such as cardiac disease and surgical fields. Many specialty hospitals advertise high rates of patient satisfaction. ${ }^{7-11}$ However, specialty hospitals differ from GMHs in significant ways. Patients at specialty hospitals may be less severely $\mathrm{ill}^{10,12}$ and may have more generous insurance coverage. ${ }^{13}$ Many specialty hospitals do not have an emergency department (ED), and their outcomes may reflect care of relatively stable patients. ${ }^{14}$ A significant number of the specialty hospitals are physician-owned, which may provide an opportunity for physicians to deliver more patient-focused healthcare. ${ }^{14}$ It is also thought that specialty hospitals can provide high-quality care by designing their facilities and service structure entirely to meet the needs of a narrow set of medical conditions.

HCAHPS survey results provide an opportunity to compare satisfaction scores among various types of hospitals. We analyzed national HCAHPS data to 
compare satisfaction scores of specialty hospitals and GMHs and identify factors that may be responsible for this difference.

\section{METHODS}

This was a cross-sectional analysis of national HCAHPS survey data. The methods for administration and reporting of the HCAHPS survey have been described. ${ }^{15}$ HCAHPS patient satisfaction data and hospital characteristics, such as location, presence of an ED, and for-profit status, were obtained from Hospital Compare database. Teaching hospital status was identified using the CMS 2013 Open Payment teaching hospital listing. ${ }^{16}$

For this study, we defined specialty hospitals as acute-care hospitals that predominantly provide care in a medical or surgical specialty and do not provide care to general medical patients. Based on this definition, specialty hospitals include cardiac hospitals, orthopedic and spine hospitals, oncology hospitals, and hospitals providing multispecialty surgical and procedure-based services. Children's hospitals, longterm acute-care hospitals, and psychiatry hospitals were excluded.

Specialty hospitals were identified using hospital name searches in the HCAHPS database, the American Hospital Association 2013 Annual Survey, the Physician Hospital Association hospitals directory, and through contact with experts. The specialty hospital status of hospitals was further confirmed by checking hospital websites or by directly contacting the hospital.

We analyzed 3-year HCAHPS patient satisfaction data that included the reporting period from July 2007 to June 2010. HCAHPS data are reported for 12 -month periods at a time. Hospital information, such as address, presence of an ED, and for-profit status were obtained from the CMS Hospital Compare 2010 dataset. Teaching hospital status was identified using the CMS 2013 Open Payment teaching hospital listing. ${ }^{16}$ For the purpose of this study, scores on the HCAHPS survey item "definitely recommend the hospital" was considered to represent overall satisfaction for the hospital. This is consistent with use of this measure in other sectors in the service industry. ${ }^{17,18}$ Other survey items were considered subdomains of satisfaction. For each hospital, the simple mean of satisfaction scores for overall satisfaction and each of the subdomains for the three 12-month periods was calculated. Data were summarized using frequencies and mean \pm standard deviation. The primary dependent variable was overall satisfaction. The main independent variables were specialty hospital status (yes or no), teaching hospital status (yes or no), for-profit status (yes or no), and the presence of an ED (yes or no). Multiple linear regression analysis was used to adjust for the above-noted independent variables. A $P$ value $<0.05$ was considered significant. All analyses were performed on Stata 10.1 IC (StataCorp, College Station, TX).

\section{RESULTS}

We identified 188 specialty hospitals and 4638 GMHs within the HCAHPS dataset. Fewer specialty hospitals had emergency care services when compared with GMHs $(53.2 \%$ for specialty hospitals vs $93.6 \%$ for GMHs, $P<0.0001$ ), and $47.9 \%$ of all specialty hospitals were in states that do not require a Certificate of Need, whereas only $25 \%$ of all GMHs were present in these states. For example, Texas, which has $7.2 \%$ of all GMHs across the nation, has $24.7 \%$ of all specialty hospitals. As compared to GMHs, a majority of specialty hospitals were for profit $(14.5 \%$ vs $66.9 \%)$.

In unadjusted analyses, specialty hospitals had significantly higher patient satisfaction scores compared with GMHs. Overall satisfaction, as measured by the proportion of patients that will "definitely" recommend that hospital, was $18.8 \%$ higher for specialty hospitals than GMHs $(86.6 \%$ vs $67.8 \%, P<0.0001)$. This was also true for subdomains of satisfaction including physician communication, nursing communication, and cleanliness (Table 1 ).

We next examined the effect of survey response rate. The survey response rate for specialty hospitals was on average 17.4 percentage points higher than that of GMHs $(49.6 \%$ vs $32.2 \%, P<0.0001)$. When adjusted for survey response rate, the difference in overall satisfaction for specialty hospitals was reduced to $8.6 \%(6.7 \%-10.5 \%, P<0.0001)$. Similarly, the differences in score for subdomains of satisfaction were more modest when adjusted for higher survey response rate. In the multiple regression models, specialty hospital status, survey response rate, for-profit status, and the presence of an ED were independently associated with higher overall satisfaction, whereas teaching hospital status was not associated with overall satisfaction. Addition of for-profit status and presence of an ED in the regression model did not change our results. Further, the satisfaction subdomain scores for specialty hospitals remained significantly higher than for GMHs in the regression models (Table 1).

\section{DISCUSSION}

In this national study, we found that specialty hospitals had significantly higher overall satisfaction scores on the HCAHPS satisfaction survey. Similarly, significantly higher satisfaction was noted across all the satisfaction subdomains. We found that a large proportion of the difference between specialty hospitals and GMHs in overall satisfaction and subdomains of satisfaction could be explained by a higher survey response rate in specialty hospitals. After adjusting for survey response rate, the differences were comparatively modest, although remained statistically significant. Adjustment for additional confounding variables did not change our results. 
TABLE 1. Satisfaction Scores for Specialty Hospitals and General Medical Hospitals and Survey Response RateAdjusted Difference in Satisfaction Scores for Specialty Hospitals

\begin{tabular}{|c|c|c|c|c|c|}
\hline Satisfaction Domains & $\begin{array}{l}\text { GMH, Mean, } \\
\mathrm{n}=4,638^{*}\end{array}$ & $\begin{array}{l}\text { Specialty Hospital, } \\
\text { Mean, } n=188^{*}\end{array}$ & $\begin{array}{l}\text { Unadjusted Mean } \\
\text { Difference in } \\
\text { Satisfaction (95\% Cl) }\end{array}$ & $\begin{array}{c}\text { Mean Difference } \\
\text { in Satisfaction Adjusted } \\
\text { for Survey Response Rate }(95 \% \text { Cl) }\end{array}$ & $\begin{array}{c}\text { Mean Difference } \\
\text { in Satisfaction for } \\
\text { Full Adjusted Model }(95 \% \mathrm{Cl})^{\dagger}\end{array}$ \\
\hline Nurses "always" communicated well & $75.0 \%$ & $84.4 \%$ & $9.4 \%(8.3-10.5)^{\ddagger}$ & $4.0 \%(2.9-5.0)^{\ddagger}$ & $5.0 \%(3.8-6.2)^{\ddagger}$ \\
\hline Pain "always" well controlled & $68.7 \%$ & $77.1 \%$ & $8.6 \%(7.7-9.6)^{\ddagger}$ & $4.5 \%(3.5-4.5)^{\ddagger}$ & $4.6 \%(3.5-5.6)^{\ddagger}$ \\
\hline "Always" received help as soon as they wanted & $62.9 \%$ & $78.6 \%$ & $15.7 \%(14.1-17.4)^{\ddagger}$ & $7.8 \%(6.1-9.4)^{\ddagger}$ & $8.0 \%(6.3-9.7)^{\ddagger}$ \\
\hline Room and bathroom "always" clean & $70.1 \%$ & $81.1 \%$ & $11.0 \%(9.6-12.4)^{\ddagger}$ & $5.5 \%(4.0-6.9)^{\ddagger}$ & $6.2 \%(4.7-7.8)^{\ddagger}$ \\
\hline $\begin{array}{l}\text { Overall satisfaction (yes, patients } \\
\text { would definitely recommend the hospital) }\end{array}$ & $67.8 \%$ & $86.6 \%$ & $18.8 \%(17.0-20.6)^{\ddagger}$ & $8.5 \%(6.9-10.2)^{\ddagger}$ & $8.6 \%(6.7-10.5)^{\ddagger}$ \\
\hline Survey response rate & $32.2 \%$ & $49.6 \%$ & $17.4 \%(16.0-18.9)^{\ddagger}$ & - & - \\
\hline
\end{tabular}

NOTE: Abbreviations: Cl, confidence interval; GMH, general medical hospital, SD, standard deviation.

*Number may vary for individual items.

${ }^{\dagger}$ Adjusted for survey response rate, presence of emergency department, teaching hospital status, and for-profit status.

${ }^{\ddagger} P<0.0001$.

Studies have shown that specialty hospitals, when compared to GMHs, may treat more patients in their area of specialization, care for fewer sick and Medicaid patients, have greater physician ownership, and are less likely to have ED services. ${ }^{11-14}$ Two small studies comparing specialty hospitals to GMHs suggest that higher satisfaction with specialty hospitals was attributable to the presence of private rooms, quiet environment, accommodation for family members, and accessible, attentive, and well-trained nursing staff. ${ }^{10,11}$ Although our analysis did not account for various other hospital and patient characteristics, we expect that these factors likely play a significant role in the observed differences in patient satisfaction.

Survey response rate can be an important determinant of the validity of survey results, and a response rate $>70 \%$ is often considered desirable. ${ }^{19,20}$ However, the mean survey response rate for the HCAHPS survey was only $32.8 \%$ for all hospitals during the survey period. In the outpatient setting, a higher survey response rate has been shown to be associated with higher satisfaction rates. ${ }^{21}$ In the hospital setting, a randomized study of a HCAHPS survey for 45 hospitals found that patient mix explained the nonresponse bias. However, this study did not examine the roles of severity of illness or insurance status, which may account for the differences in satisfaction seen between specialty hospitals and GMHs. ${ }^{22}$ In contrast, we found that in the hospital setting, higher survey response rate was associated with higher patient satisfaction scores.

Our study has some limitations. First, it was not possible to determine from the dataset whether higher response rate is a result of differences in the patient population characteristics between specialty hospitals and GMHs or it represents the association between higher satisfaction and higher response rate noted by other investigators. Although we used various resources to identify all specialty hospitals, we may have missed some or misclassified others due to lack of a standardized definition. ${ }^{10,12,13}$ However, the total number of specialty hospitals and their distribution across various states in the current study are consistent with previous studies, supporting our belief that few, if any, hospitals were misclassified. ${ }^{13}$

In summary, we found significant difference in satisfaction rates reported on HCAHPS in a national study of patients attending specialty hospitals versus GMHs. However, the observed differences in satisfaction scores were sensitive to differences in survey response rates among hospitals. Teaching hospital status, forprofit status, and the presence of an ED did not appear to further explain the differences. Additional studies incorporating other hospital and patient characteristics are needed to fully understand factors associated with differences in the observed patient satisfaction between specialty hospitals and GMHs. Additionally, strategies to increase survey HCAHPS response rates should be a priority.

\section{References}

1. About Picker Institute. Available at: http://pickerinstitute.org/about. Accessed September 24, 2012.

2. HCAHPS Hospital Survey. Centers for Medicare \& Medicaid Services, Baltimore, MD. Available at: http://www.hcahpsonline.org. Accessed September 24, 2012.

3. Survey of patients' hospital experiences (HCAHPS). Centers for Medicare \& Medicaid Services, Baltimore, MD. Available at: http://data. medicare.gov/dataset/Survey-of-Patients-Hospital-Experiences-HCAHPS/rj76-22dk. Accessed August 24, 2012.

4. Isaac T, Zaslavsky AM, Cleary PD, Landon BE. The relationship between patients' perception of care and measures of hospital quality and safety. Health Serv Res. 2010;45(4):1024-1040.

5. Huppertz JW, Carlson JP. Consumers' use of HCAHPS ratings and word-of-mouth in hospital choice. Health Serv Res. 2010;45(6 pt 1): 1602-1613.

6. Otani K, Herrmann PA, Kurz RS. Improving patient satisfaction in hospital care settings. Health Serv Manage Res. 2011;24(4):163-169.

7. Live the life you want. Arkansas Surgical Hospital website. Available at: http://www.arksurgicalhospital.com/ash. Accessed September 24, 2012. 
8. Patient satisfaction-top 60 hospitals. Hoag Orthopedic Institute website. Available at: http://orthopedichospital.com/2012/06/patientsatisfaction-top-60-hospital. Accessed September 24, 2012.

9. Northwest Specialty Hospital website. Available at: http://www.northwestspecialtyhospital.com/our-services. Accessed September 24, 2012.

10. Greenwald L, Cromwell J, Adamache W, et al. Specialty versus community hospitals: referrals, quality, and community benefits. Health Affairs. 2006;25(1):106-118.

11. Study of Physician-Owned Specialty Hospitals Required in Section 507(c)(2) of the Medicare Prescription Drug, Improvement, and Modernization Act of 2003, May 2005. Available at: http://www.cms.gov/Medi care/Fraud-and-Abuse/PhysicianSelfReferral/Downloads/RTC-Studyof PhysOwnedSpecHosp.pdf. Accessed June 16, 2014.

12. Specialty Hospitals: Information on National Market Share, Physician Ownership and Patients Served. GAO: 03-683R. Washington, DC: General Accounting Office; 2003:1-20. Available at: http:// www.gao.gov/new.items/d03683r.pdf. Accessed September 24, 2012.

13. Cram P, Pham HH, Bayman L, Vaughan-Sarrazin MS. Insurance status of patients admitted to specialty cardiac and competing general hospitals: are accusations of cherry picking justified? Med Care. 2008;46: $467-475$.

14. Specialty Hospitals: Geographic Location, Services Provided and Financial Performance: GAO-04-167. Washington, DC: General Accounting Office; 2003:1-41. Available at: http://www.gao.gov/ new.items/d04167.pdf. Accessed September 24, 2012.
15. Centers for Medicare \& Medicaid Services. HCAHPS quality assurance guidelines. Version 8.0. Available at: http://www.hcahpsonline. org/Files/HCAHPS\%20QAG\%20V8.0\%20MARCH\%202013.pdf. Accessed June 6, 2014.

16. Teaching hospitals. Centers for Medicare \& Medicaid Services. Available at: http://www.cms.gov/Regulations-and-Guidance/Legislation/ National-Physician-Payment-Transparency-Program/Teaching-Hospitals. html. Accessed December 30, 2013.

17. Woodside AG, Frey LL, Daly RT. Linking service quality, customer satisfaction and behavioral intention. J Health Care Mark. 1989;9(4): 5-17.

18. Gronholdt L, Martensen A, Kristensen K. The relationship between customer satisfaction and loyalty: cross-industry differences. Total Oual Manage. 2000;11(4-6):509-514.

19. Baruch Y, Holtom BC. Survey response rate levels and trends in organizational research. Hum Relat. 2008;61:1139-1160.

20. Machin D, Campbell MJ. Survey, cohort and case-control studies. In: Design of Studies for Medical Research. Hoboken, NJ: John Wiley \& Sons Ltd.; 2005:118-120.

21. Mazor KM, Clauser BE, Field T, Yood RA, Gurwitz JH. A demonstration of the impact of response bias on the results of patient satisfaction surveys. Health Serv Res. 2002;37(5):1403-1417.

22. Elliott M, Zaslavsky A, Goldstein E, et al. Effects of survey mode, patient mix and nonresponse on CAHPS hospital survey scores. Health Serv Res. 2009;44:501-518. 